\section{Commentary: What's ahead for cardiac surgery-associated acute kidney injury after discharge}

\author{
Natasha Afonso, MD, MPH, and \\ Paul A. Checchia, MD, FCCM, FACC
}

Cardiac surgery-associated acute kidney injury (CS-AKI) often results in poor outcomes with increased morbidity and mortality in adult and pediatric patients. ${ }^{1,2}$ The effect of CS-AKI with postdischarge outcomes is explored by Nunes and colleagues ${ }^{3}$ in their large multicenter prospective study reported in this issue of the Journal. The authors examine short- and long-term outcomes after pediatric cardiac surgery and demonstrate the increased risk of 30-day mortality in patients with severe CS-AKI. Their article adds to the growing body of evidence demonstrating the deleterious impact of CS-AKI on our patients.

It is important to note that diagnosis of AKI is based on serum creatinine levels, which can vary based on age, sex, muscle mass, and fluid status. Furthermore, delays in diagnosis often occur, because an increase in serum creatinine level is not apparent until the glomerular filtration rate declines by $50 \%$. Changes in serum creatinine also do not predict whether kidney function is irreversibly damaged or will improve with time. Although the authors did not find a relationship between CS-AKI and hospital readmission rate, the absence of evidence may be related in part to the inherent limitations in assessing AKI as described above. This article highlights the importance of accurate and early diagnosis of AKI to initiate potential therapy and mitigate long-term renal injury.

From the Section of Critical Care Medicine, Department of Pediatrics, Texas Children's Hospital, Baylor College of Medicine, Houston, Tex.

Disclosures: The authors reported no conflicts of interest.

The Journal policy requires editors and reviewers to disclose conflicts of interest and to decline handling or reviewing manuscripts for which they may have a conflict of interest. The editors and reviewers of this article have no conflicts of interest.

Received for publication Aug 20, 2020; revisions received Aug 20, 2020; accepted for publication Aug 21, 2020; available ahead of print Sept 17, 2020.

Address for reprints: Paul A. Checchia, MD, FCCM, FACC, Section of Critical Care Medicine, Department of Pediatrics, Texas Children's Hospital, Baylor College of Medicine, 6651 Main St, Houston, TX 77030 (E-mail: checchia@bcm.edu).

JTCVS Open 2020;4:86-7

2666-2736

Copyright (C) 2020 The Authors. Published by Elsevier Inc. on behalf of The American Association for Thoracic Surgery. This is an open access article under the CC BY-NCND license (http://creativecommons.org/licenses/by-nc-nd/4.0/).

https://doi.org/10.1016/j.xjon.2020.08.011

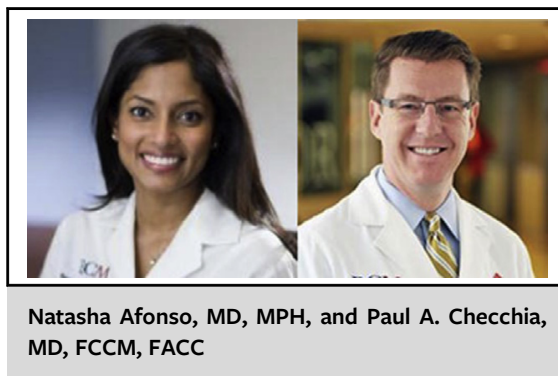

CENTRAL MESSAGE

Acute kidney injury (AKI)

following pediatric cardiac sur-

gery is an increasingly recognized

concern with important implica-

tions on prognosis and manage-

ment. Improved diagnostic

methods are on the horizon to

identify AKI early and new treatment technologies that can help

mitigate long-term effects.

Novel biomarkers have been identified in the early stress response of the kidney to AKI and appear in urine or plasma well before a change in serum creatinine level is detected. Many of these are being developed and validated to identify AKI earlier and to predict severity. ${ }^{4}$ In the future, we may see better stratification of renal damage and injury as we incorporate other biomarkers, such as cystatin $\mathrm{C}$ and neutrophil gelatinase-associated lipocalin, both of which have been shown to represent earlier markers of AKI and recovery than serum creatinine. ${ }^{5-7}$

Early interventions to treat CS-AKI include the avoiding nephrotoxins and managing fluid overload. If dialysis is required, early initiation may lead to improved clinical outcomes. For neonates, peritoneal dialysis has traditionally been the modality of choice but is not always available or effective, such as in patients with previous abdominal surgeries or infections or in patients who require more efficient solute clearance. Dialysis via a hemocatheter also has challenges in small infants, owing primarily to difficulties in access. In addition, the large relative extracorporeal volume may necessitate blood product exposure for priming in small patients. Emerging technologies will expand the therapies offered for the smallest of patients affected with AKI, however. One example is the recent introduction of a miniaturized cardio-renal pediatric dialysis emergency machine $\left(\right.$ CARPEDIEM) ${ }^{8}$ for infants weighing $<10 \mathrm{~kg}$. 
This machine requires a priming volume of $<30 \mathrm{~mL}$ with low flow rates, allowing for easier dialysis in smaller children.

Investigations on how to best identify, prevent, and treat CS-AKI in pediatric patients are ongoing. We look forward to the improved diagnostic and therapeutic strategies to help mitigate CS-AKI-associated mortality and morbidity.

\section{References}

1. Li S, Krawczeski CD, Zappitelli M, Devarajan P, Thiessen-Philbrook H, Coca SG, et al. Incidence, risk factors, and outcomes of acute kidney injury after pediatric cardiac surgery: a prospective multicenter study. Crit Care Med. 2011;39:1493-9.

2. Chertow GM, Levy EM, Hammermeister KE, Grover F, Daley J. Independent association between acute renal failure and mortality following cardiac surgery. Am J Med. 1998;104:343-8.
3. Nunes S, Brown J, Parikh CR, Greenberg JH, Devarajan P, Philbrook HT, et al. The association of acute kidney injury with hospital readmission and death after pediatric cardiac surgery. J Thorac Cardiovasc Surg Open. 2020;4:70-85.

4. Krawczeski CD, Goldstein SL, Woo JG, Wang Y, Piyaphanee N, Ma Q, et al. Temporal relationship and predictive value of urinary acute kidney injury biomarkers after pediatric cardiopulmonary bypass. J Am Coll Cardiol. 2011;58:2301-9.

5. Lau L, Al-Ismaili Z, Harel-Sterling M, Pizzi M, Caldwell JS, Piccioni M, et al Serum cystatin C for acute kidney injury evaluation in children treated with aminoglycosides. Pediatr Nephrol. 2017;32:163-71.

6. Alge JL, Arthur JM. Biomarkers of AKI: a review of mechanistic relevance and potential therapeutic implications. Clin J Am Soc Nephrol. 2015;10:147-55.

7. Zhou F, Luo Q, Wang L, Han L. Diagnostic value of neutrophil gelatinaseassociated lipocalin for early diagnosis of cardiac surgery-associated acute kidney injury: a meta-analysis. Eur J Cardiothorac Surg. 2016;49:746-55.

8. Ronco DC, Garzotto F, Brendolan A, Zanella M, Bellettato M, Vedovato S, et al. Continuous renal replacement therapy in neonates and small infants: development and first-in-human use of a miniaturised machine (CARPEDIEM). Lancet. 2014; $383: 1807-13$. 